\title{
Particle Filter based Autonomous Underwater Vehicle Navigation System aided thru acoustic communication ranging
}

\author{
Bruno V. Menna \\ Hydroacoustics Cabinet \\ National Institute for Fisheries Research and \\ Development (INIDEP) \\ Mar del Plata, Argentina \\ bmenna@inidep.edu.ar
}

\author{
Sebastian A. Villar \\ INTELYMEC-CIFICEN (UNCPBA, \\ CICPBA, CONICET) \\ FIO, UNCPBA \\ Olavarría, Argentina \\ svillar@fio.unicen.edu.ar
}

\author{
Gerardo G. Acosta \\ INTELYMEC-CIFICEN (UNCPBA, \\ CICPBA, CONICET) \\ FIO, UNCPBA \\ Olavarría, Argentina \\ ggacosta@fio.unicen.edu.ar
}

\begin{abstract}
Autonomous Underwater Vehicles (AUVs) are platforms suitable for a wide variety of applications in the marine environment with economic and operational advantages. In these applications an AUV performs a given task as a mission. During the mission execution, the AUV will move around the environment following paths that allow it to fulfill the mission's objectives. To achieve this, a reliable Navigation System (NS) is required. In addition to this, the current operating concept includes the deployment of multiple AUVs on a given area, thus a communication system between vehicles is also required. In the underwater environment both navigation and communication systems deals with the particular characteristics of the medium that limits the use of conventional techniques. In this work, a complete NS for an AUV is presented. The developed NS is based on an inertial navigation scheme with velocity and position aiding. The position aiding takes advantage of the communication system onboard the vehicle, which avoids the use of additional positioning systems. The fundamentals of the applied solutions are described and experimental results and implementation details are provided. Also conclusions and future works are presented.
\end{abstract}

Keywords-Autonomous Underwater Vehicles, Navigation System, Particle Filter, Particle Swarm Optimization, Robot Operating System

\section{INTRODUCTION}

Autonomous Underwater Vehicles (AUVs) are platforms suitable for a wide variety of applications in several fields in the marine environment with economic and operational advantages [1], [2] and [3].

In any of these applications an AUV performs a given task as a mission, that consists on a plan with specific objectives. During the mission execution, the AUV will move around the environment following paths that allow it to fulfill the mission's objectives. To achieve this, a reliable Navigation, Guidance and Control (NGC) structure is required [4]. This NGC structure is composed basically by; Navigation System (NS) that provides information about the speed, position and attitude of the vehicle, Guidance system (GS) responsible for establishing the position and speed set-points to develop the required trajectory and the Control system (CS) formed by the control loops to command the vehicle's propulsion system. The NS information is also necessary to locate the data acquired by the AUV payload sensors.

Recently, multiple surface and underwater autonomous vehicles in coordinated operation arises as a flexible, reliable and efficient operation scheme. Communication between vehicles is required in order to allow coordinated operation. Acoustic carriers are the more reliable way to implement an underwater communication. This scenario constitutes an UnderWater Acoustic Sensor Network (UWASN) [5].

A NS provides information about the velocity, position and attitude of a vehicle. Within this definition one of the components of a NS is the Positioning System (PS) that provides position and attitude information.

The underwater environment imposes particular challenges for the development of navigation and positioning solutions. The main limitation is the impossibility of using Global Position System (GPS) devices. Despite this, given the good propagation characteristics of the acoustics waves in the underwater medium, several acoustic positioning and navigation devices have been developed.

Acoustic positioning systems are the Long BaseLine (LBL), UltraShort BaseLine (USBL) and GPS Intelligent Buoys (GIB) [6] and [7]. These systems have proven to be accurate, but requiring the deployment of an additional infrastructure and the integration of specific devices onboard the vehicles. This may not be possible in particular environments, such as polar regions, or by budget or technical restrictions in certain vehicles. Then the need arises to develop positioning solutions that require minimal additional infrastructure and devices onboard the vehicles.

The limitation of GPS reception in the underwater medium also difficult the vehicles velocity measurement to develop a navigation system. The most widely used device to perform underwater velocity measurement is the Doppler Velocity Log (DVL), an acoustic device that measures the vehicles velocity with respect to the sea bottom [8]. The downside of this device is its high cost, a promising low cost alternative is the development of a pressure based velocity sensor for underwater applications which has shown good results in its first tests [9]. 
Given velocity and heading measurements at known time intervals, a navigation technique named Dead Reckoning (DR) might be implemented to estimate the vehicles position [10].

Inertial Navigation Systems (INS) have some advantages over DR, allowing to estimate the velocity and position of a vehicle at higher update rates $(>50 \mathrm{~Hz})$ from acceleration and attitude measurements. The implementation of an INS has low computational cost. However, one of its main problems is the accumulation of velocity and position estimation errors. This is because the velocity and position estimates are performed by successive acceleration integrations [11]. In some cases, such as in short duration missions or in shallow waters, those errors are corrected by surfacing the vehicle to acquire GPS measurements [12]. But the most reliable way to compensate the estimation errors in an INS is the use of aiding measurements from additional sensors. Aiding measurements are complementary to the INS estimates, in the sense that the lasts are more frequent and accurate in the short term and the previous are generally less frequent but accurate in the long term.

On an Aided INS (AINS) the estimates are corrected based on the error between the INS estimates and the measurements from the aiding sensors through estimation algorithms. These estimation algorithms are based in Bayesian filtering techniques [13] such as the Kalman Filter (KF). Given the nonlinearity of the aided INS problem, variants of the KF are applied, including the Extended Kalman Filter (EKF) and Unscented Kalman Filter (UKF). Several implementations of underwater AINS based on the EKF algorithm have been developed with good results [14], [15] and [16]. Less UKF implementations exists but also with satisfactory results [17]. A non-parametric Bayesian filtering algorithm that allows to treat non-linear and non-Gaussian systems is the Particle Filter (PF) algorithm. The PF has the potential to provide better results than the EKF or UKF implementations [18] and [19]. Despite this, PF based underwater navigation implementations are scarce [20]. One of the drawbacks of the PF is its higher computational cost compared to an EKF or UKF. Although at present it is possible to overcome this limitation with the modern compact and high performance computing units suitable for be employed in underwater vehicles.

In this work the development of a NS for an AUV is presented. The system considers the development of an underwater PS employing the usual sensors onboard an AUV, thus requiring minimum additional infrastructure. The NS itself is based on an AINS that employs the PF as estimation algorithm. This work is organized as follow; in Section II the relevant theoretical aspects are described, in Section III the developed navigation and positioning solutions are detailed, in Section IV implementation details and preliminary results are presented and in Section V conclusions are discussed.

\section{THEORETICAL FUNDAMENTALS}

In this section the main theoretical aspects of this work are presented; inertial navigation, aided inertial navigation and state estimation.

\section{A. Inertial Navigation}

Inertial navigation is based on the laws of classical mechanics. So, by measuring the accelerations of the vehicle it is possible to determine its velocity by integrating the acceleration with respect to time. Similarly, the change in the vehicle position is determined by integrating the velocity with respect to time.

Accelerations are measured by a triplet of sensors called accelerometers oriented on mutually perpendicular XYZ axes that constitute the vehicles reference frame, also called body frame (b-frame).

To estimate the vehicles velocity and position in the geographic reference frame ( $n$-frame) of axes NED (North, East, Down) the vehicle attitude is required. The $n$-frame is defined by a plane tangent to the Earth surface at latitude $L$ and longitude $l$ both measured from an Earth centered frame (e-frame). These reference frames are represented in Fig. 1.

The inertial navigation computation starts with the transformation of the vehicle acceleration measurements vector $\boldsymbol{f}^{b}$ from the $b$-frame to the $n$-frame through a Direction Cosines Matrix (DCM) $\boldsymbol{C}_{b}^{n}$ :

$$
\boldsymbol{f}^{n}=\left[\begin{array}{l}
f_{N} \\
f_{E} \\
f_{D}
\end{array}\right]=\boldsymbol{C}_{b}^{n} \boldsymbol{f}^{b}=\boldsymbol{C}_{b}^{n}\left[\begin{array}{l}
f_{X} \\
f_{Y} \\
f_{Z}
\end{array}\right]
$$

where; $\boldsymbol{f}^{n}$ is the acceleration measurements vector in the $n$ frame and $f_{N}, f_{E}$ and $f_{D}$ are the North, East and Down acceleration components. The DCM is computed from the vehicle attitude measurements, as seen in equation (2), where; $q_{W}, q_{X}, q_{Y}$ and $q_{Z}$ are the attitude components, expressed in quaternions.

The DCM is an ortho-normal matrix. The orthogonality and normality is maintained by correction at every computation [21].

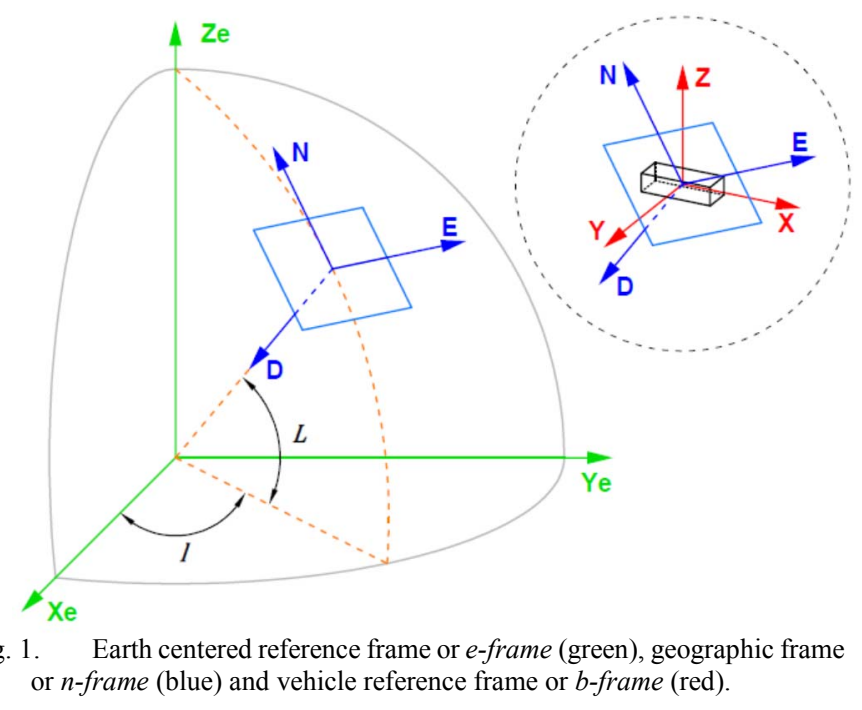




$$
\boldsymbol{C}_{b}^{n}=\left[\begin{array}{ccc}
\left(q_{W}^{2}+q_{X}^{2}-q_{Y}^{2}-q_{Z}^{2}\right) & 2\left(q_{X} q_{Y}-q_{W} q_{Z}\right) & 2\left(q_{X} q_{Z}+q_{W} q_{Y}\right) \\
2\left(q_{X} q_{Y}+q_{W} q_{Z}\right) & \left(q_{W}^{2}-q_{X}^{2}+q_{Y}^{2}-q_{Z}^{2}\right) & 2\left(q_{Y} q_{Z}-q_{W} q_{X}\right) \\
2\left(q_{X} q_{Z}-q_{W} q_{Y}\right) & 2\left(q_{Y} q_{Z}+q_{W} q_{X}\right) & \left(q_{W}^{2}-q_{X}^{2}-q_{Y}^{2}+q_{Z}^{2}\right)
\end{array}\right]
$$

Assuming the Earth shape as spherical, the vehicle velocity change in the $n$-frame $\dot{\boldsymbol{V}}^{n}$ is estimated by subtracting the gravity $\left(\boldsymbol{g}_{l}^{n}\right)$, Coriolis and centripetal $\left(\boldsymbol{f}_{C}^{n}\right)$ accelerations [11]:

$$
\dot{\boldsymbol{V}}^{n}=\left[\begin{array}{c}
\dot{V}_{N} \\
\dot{V}_{E} \\
\dot{V}_{D}
\end{array}\right]=\boldsymbol{f}^{n}-\boldsymbol{f}_{C}^{n}+\boldsymbol{g}_{l}^{n}
$$

where; $\dot{V}_{N}, \dot{V}_{E}$ and $\dot{V}_{D}$ al tiu ciranges of velocity in the North, East and Down directions of the $n$-frame.

Then, the vehicle velocity in the $n$-frame is estimated by integration of the results from equation (3).

The vehicle position change in the $n$-frame $\dot{\boldsymbol{P}}^{n}$ is estimated as follow:

$$
\dot{\boldsymbol{P}}^{n}=\left[\begin{array}{c}
\dot{L} \\
\dot{l} \\
\dot{h}
\end{array}\right]=\left[\begin{array}{c}
V_{N} /\left(R_{0}+h\right) \\
V_{E} \sec (L) /\left(R_{0}+h\right) \\
-V_{D}
\end{array}\right]
$$

where; $R_{0}$ is the Earth radius (6378137 m), $\dot{L}, \dot{l}$ and $\dot{h}$ are the changes in latitude, longitude and altitude (or depth) respectively.

Then, the vehicle position in the $n$-frame is estimated by integration of the results from equation (4).

The velocity and position estimates obtained from the inertial navigation computation are grouped in a navigation state vector $\boldsymbol{x}_{I N \boldsymbol{S}}$.

$$
\boldsymbol{x}_{I N \boldsymbol{S}}=\left[\begin{array}{l}
\boldsymbol{V}^{n} \\
\boldsymbol{P}^{n}
\end{array}\right]=\left[\begin{array}{llllll}
V_{N} & V_{E} & V_{D} & L & l & h
\end{array}\right]^{T}
$$

As can be seen, the inertial navigation computation requires measurements of both accelerations and attitude and is not a complex process. However, due to the double integration the measurement errors in accelerations and attitude accumulate in velocity and position estimates, thus resulting in increasing and unbounded estimation errors.

\section{B. Aided Inertial Navigation and State Estimation}

An alternative to overcome the error accumulation on an INS consists in the use of additional sensors measurements, usually called aiding measurements. This kind of system constitutes an Aided Inertial Navigation System (AINS).

In an AINS, the estimates from the INS are converted by a function to equivalents magnitudes to those of the aiding sensors. The differences between the aiding measurements and the estimated measurements are used in an estimation algorithm to obtain an improved estimation of the navigation states.

The aiding measurements are less frequent but accurate in the long term while the inertial navigation estimates are more frequent but accurate in the short term. So, in an AINS the inertial estimates are updated periodically when an aiding measurement is available and in absence of aiding measurements the states are estimated by the inertial navigation.
Estimation algorithms applied to AINS are mostly based in Bayesian filtering schemes. Within this schemes, the Particle Filter (PF) is a non-parametric Bayesian filter. As EKF or UKF the PF is a suboptimal estimator. All the PF, EKF or UKF allow to estimate non-linear systems states, but the PF also allows to estimate the real Probability Density Function (PDF) of the states, while the EKF and the UKF assumes a Gaussian PDF [13].

In the PF algorithm, a set of states, or particles, are generated from a given distribution (a uniform distribution, for example). Every particle in the set has an associated weight that represents the probability of the particle to represent the real state of the system.

Initially all the particles have the same weight, so all has the same probability to represent the real state of the system.

The following values of the particles are predicted trough the INS computation, and its weights are no modified.

But, when an aiding measurement is available the particles weights are updated through a likelihood function between the aiding measurement and the estimated measurement computed from the particles. This results in that some particles preserve a relative high weight, thus representing the most probable values of the real states of the system, while others will have a lower weight, thus representing the less probable values of the real states of the system. In this way, the PF allows to represent the real PDF of the system states.

However, after some updates the majority of the particles weights becomes negligible. This is a problem, because the particles with negligible weight will not provide information to the estimation. To avoid this, after every update, the effective particle number of the particle set is calculated. If this effective weight is less than a threshold the particle set is re-sampled [22]. Through the re-sampling process, the particles with lower weight are suppressed and those with higher weight are reproduced, as an analogy of the "survival of the fittest" principle. There are several re-sampling algorithms [23], such as the systematic re-sampling and the selection and resampling [24] and [25]. The PF algorithm might be divided into five stages:

1) Initialization: in this stage an initial set $\boldsymbol{X}_{0}$ of $N$ particles $\boldsymbol{x}_{0}^{i}$ from an uniform distribution in the state space is created. The weight $w_{0}^{i}$ assigned to all particles is equal to $1 / N$.

$$
\begin{gathered}
\boldsymbol{X}_{0}=\left[\boldsymbol{x}_{0}^{i}\right] \text { with } i=1,2, \ldots N \\
\boldsymbol{W}_{0}=\left[w_{0}^{i}\right] \text { with } w_{0}^{i}=1 / N \forall i
\end{gathered}
$$

2) Prediction: the particles are propagated to the $k$ instant through a procces function $\boldsymbol{f}$ and the control inputs $\boldsymbol{u}_{k}$.

$$
\boldsymbol{X}_{k}=\boldsymbol{f}\left(\boldsymbol{x}_{k-1}^{i}, \boldsymbol{u}_{k}\right)
$$


3) Update: if at the instant $k$ an aiding measurement $\boldsymbol{z}_{k}$ is available, the weights of the $N$ are updated through a likelihood function $g$ whose argument is the difference between $\boldsymbol{z}_{k}$ and the predicted measurement $\boldsymbol{h}\left(\boldsymbol{x}_{k}^{i}\right)$ for every particle. The updated weights are the normalized.

$$
\begin{gathered}
w_{k}^{i}=w_{k-1}^{i} g\left(\boldsymbol{z}_{k}-\boldsymbol{h}\left(\boldsymbol{x}_{k}^{i}\right)\right) \\
w_{k}^{i}=\frac{w_{k}^{i}}{\sum_{i=1}^{N} w_{k}^{i}}
\end{gathered}
$$

4) $\boldsymbol{R e}$-sampling: the re-sampling stage is executed if the effective particle number $N_{e f}$ is lower than a threshold $N_{t h}$.

$$
N_{e f}=\frac{1}{\sum_{i=1}^{N}\left(w_{k}^{i}\right)^{2}}<N_{t h}
$$

After the re-sampling process, all the particles are assigned the same weight $1 / N$.

5) Estimation: the state estimation $\boldsymbol{x}_{k}$ is performed by the weighted mean of the particles by their weights.

$$
\boldsymbol{x}_{k}=\sum_{i=1}^{N} w_{k}^{i} \boldsymbol{x}_{k}^{i}
$$

After stage 5, a new iteration is carried out from stage 2.

\section{DEVELOPED SOLUTIONS}

In this section the development of the underwater navigation and positioning systems are detailed. In both systems PF estimation algorithms are applied with different variations in each case.

The underwater navigation system is based in an INS scheme aided in both velocity and position. The INS requires acceleration and attitude measurements, acceleration is obtained from an Inertial Measurement Unit (IMU) and attitude information is provided by the positioning system. Velocity aiding is provided by a Doppler Velocity Log (DVL), while position aiding is provided by the spatial information of the positioning system.

As can be seen, besides comnutation and actimation algorithms the developmen of both navigation and positioning systems also requires of a variety of sensors ancievices onboard the AUV:

- Inertial Measurement Unit (IMU)

- Doppler Velocity Log (DVL)

- Global Position System (GPS)

- Depth meter

- Acoustic modems

An additional device is also required to provide a known reference position and range measurement when AUV is submerged. In this work this device consists in a surface buoy equipped with a GPS receiver and an acoustic modem that periodically broadcast a positioning report with its GPS position. The range between the surface buoy and the AUV is estimated thru measurement of the acoustic communication Time Of
Flight (TOF). In order to perform the TOF measurement the clocks in the surface buoy and AUV must be synchronized [19].

In this way the developed systems require of a minimal additional infrastructure (surface buoy) and the normal sensors and devices integrated in an AUV.

\section{A. Underwater Navigation System}

The core of the developed underwater navigation system is an INS. The INS velocity and position estimates are aided by measurements from a DVL and the position information provided by the positioning systems. Two PF estimation algorithms were applied, one for the velocity estimation and the other for position estimation.

Initu lization: in both velocity and position PFs a set of $N_{v}$ an $N_{v}$ p articles representing probable velocity and position state a generated and an equal weight is assigned to every particle component. In the velocity PF:

$$
\begin{gathered}
\boldsymbol{v}_{j}^{0}=\left[\begin{array}{ccc}
v_{N j}^{0} & v_{E j}^{0} & v_{D j}^{0}
\end{array}\right] \operatorname{con} j=1, \ldots, N_{v} \\
\boldsymbol{w}_{v j}^{0}=\left[\begin{array}{lll}
1 / N_{v} & 1 / N_{v} & 1 / N_{v}
\end{array}\right] \forall j
\end{gathered}
$$

And in the position PF:

$$
\begin{gathered}
\boldsymbol{p}_{j}^{0}=\left[\begin{array}{lll}
L_{j}^{0} & l_{j}^{0} & z_{j}^{0}
\end{array}\right] \operatorname{con} j=1, \ldots, N_{p} \\
\boldsymbol{w}_{p j}^{0}=\left[\begin{array}{lll}
1 / N_{p} & 1 / N_{p} & 1 / N_{p}
\end{array}\right] \forall j
\end{gathered}
$$

Prediction: estate prediction in both velocity and position PFs is based in the INS computation (Section II-A).

For velocity prediction, accelerometer measurements (referred to the $b$-frame) at the instant $k \boldsymbol{a}_{b}^{k}$ are translated to the $n$-frame by means of the DCM $\boldsymbol{C}_{b}^{n}(k)$ :

$$
\boldsymbol{a}_{n}^{k}=\boldsymbol{C}_{b}^{n}(k) \boldsymbol{a}_{b}^{k}
$$

With the acceleration measurements now referred to the nframe $\boldsymbol{a}_{n}^{k}$, the velocity change $\dot{\boldsymbol{v}}_{j}^{k}$ for the $j$ particle is obtained by subtraction of the Coriolis, centripetal and gravity accelerations, expressed by $\boldsymbol{a}_{C j}^{k}$ and $\boldsymbol{g}^{k}$ (detailed description in [11]).

$$
\dot{\boldsymbol{v}}_{j}^{k}=\boldsymbol{a}_{n}^{k}-\boldsymbol{a}_{C j}^{k}+\boldsymbol{g}^{k}
$$

The velocity of the $j$ particle predicted at the instant $(k+1)$ is given by integration of the velocity change $\dot{\boldsymbol{v}}_{j}^{k}$ considering the sampling time $T_{S}$ :

$$
\boldsymbol{v}_{j}^{(k+1)}=\boldsymbol{v}_{j}^{k}+T_{S} \dot{\boldsymbol{v}}_{j}^{k} \text { on } j=1, \ldots, N_{v}
$$

Position change $\dot{\boldsymbol{p}}_{j}^{k}$ for the $j$ particle at the instant $k$ is given by the estimations of the velocity $\mathrm{PF}\left(V_{N A U V}^{k}, V_{E A U V}^{k}\right.$ and $\left.V_{D A U V}^{k}\right)$ and the last depth estimation $z_{j}^{k}$ :

$$
\dot{\boldsymbol{p}}_{j}^{k}=\left[\begin{array}{c}
V_{N A U V}^{k} /\left(R_{0}+z_{j}^{k}\right) \\
V_{E A U V}^{k} \sec \left(L_{j}^{k}\right) /\left(R_{0}+z_{j}^{k}\right) \\
-V_{D A U V}^{k}
\end{array}\right]^{T}
$$

Then the position of the $j$ particle predicted at the instant $(k+1)$ is given by integration of the position change $\dot{\boldsymbol{p}}_{j}^{k}$ considering the sampling time $T_{S}$ : 


$$
\boldsymbol{p}_{j}^{(k+1)}=\boldsymbol{p}_{j}^{k}+T_{s} \dot{\boldsymbol{p}}_{j}^{k} \text { con } j=1, \ldots, N_{p}
$$

Update: whenever a velocity or position aiding measurement is available the weights of the particles in the velocity PF or position PF are updated.

The velocity PF update is carried out if a vehicle velocity measurement is available from GPS (vehicle at surface) or DVL (vehicle submerged), the difference between the aiding measurement and every particle estimated velocity is calculated:

$$
\begin{gathered}
\boldsymbol{\delta} \boldsymbol{v}^{k}=\boldsymbol{v}_{G P S / D V L}^{(k+1)}-\boldsymbol{v}_{j}^{(k+1)} \\
\boldsymbol{\delta} \boldsymbol{v}^{k}=\left[\begin{array}{lll}
\delta v_{N j}^{k+1} & \delta v_{E j}^{k+1} & \delta v_{D j}^{k+1}
\end{array}\right]
\end{gathered}
$$

The particles components weights are updated thru a likelihood function:

$$
w_{i j}^{k+1}=\frac{e^{\frac{-\left(\delta v_{i j}^{k}\right)^{2}}{2 \sigma_{\delta v}^{2}}}}{\sigma_{\delta v} \sqrt{2 \pi}} w_{i j}^{k}
$$

The updated weights are then normalized (equation (10)).

For the position PF, the update is performed when a measurement from the Positioning System (PS) is available from which the difference with the particles estimated positions is calculated:

$$
\begin{gathered}
\boldsymbol{\delta} \boldsymbol{p}^{k}=\boldsymbol{p}_{P S}^{(k+1)}-\boldsymbol{p}_{j}^{(k+1)} \\
\delta \boldsymbol{p}^{k}=\left[\begin{array}{lll}
\delta L_{j}^{k+1} & \delta l_{j}^{k+1} & \delta z_{j}^{k+1}
\end{array}\right]
\end{gathered}
$$

The particles components weights are updated thru a likelihood function:

$$
w_{i j}^{k+1}=\frac{e^{\frac{-\left(\delta p_{i j}^{k}\right)^{2}}{2 \sigma_{\delta p}^{2}}}}{\sigma_{\delta p} \sqrt{2 \pi}} w_{i j}^{k}
$$

The updated weights are then normalized.

Re-sampling: after the update stage the effective number of the particles components is calculated.

For the $i$ component of the velocity or position PFs particles, the effective particle numbers are calculated as in equation (11).

In any case if the effective particle number is lower than a threshold the re-sampling is performed. In both PFs the selection and replacement algorithm was applied.

Estimation: in both PFs velocity and position components are estimated as the weighted average of the particles components as shown in equations (26) and (27).

\section{B. Underwater Positioning System}

In this sub-section the developed AUV positioning solutions are detailed. The positioning system provides both angular and spatial position. Angular or attitude is used in INS computation (see equations (1) and (2)) and spatial position serves as aiding measurement in the AINS solution.

\section{1) Attitude estimation}

The vehicles attitude is determined from the IMU sensors measurements and expressed in quaternions.

Initially attitude is predicted by integration of the gyroscope measurements:

$$
\boldsymbol{q}_{(k)}^{g}=\frac{\boldsymbol{q}_{(k-1)}^{g}+0.5 T_{S}\left(\boldsymbol{q}_{(k-1)}^{g} \otimes \boldsymbol{q}^{\omega}\right)}{\left\|\boldsymbol{q}_{(k)}^{g}\right\|}
$$

where; $\boldsymbol{q}_{(k)}^{g}$ and $\boldsymbol{q}_{(k-1)}^{g}$ are the attitude predictions from the gyroscope measurement a he instants $(k)$ and $(k-1), \otimes$ denotes the quaternion product operator, $T_{S}$ is the sampling period (s) and $\boldsymbol{q}^{\omega}$ is the angular velocity measurements vector $(\mathrm{rad} / \mathrm{s})$.

$$
\boldsymbol{q}^{\omega}=\left[\begin{array}{ll}
0 & \boldsymbol{\omega}_{b}
\end{array}\right]^{T}=\left[\begin{array}{llll}
0 & \omega_{X} & \omega_{Y} & \omega_{Z}
\end{array}\right]^{T}
$$

where; $\boldsymbol{\omega}_{b}=\left[\begin{array}{lll}\omega_{X} & \omega_{Y} & \omega_{Z}\end{array}\right]$ is the gyroscope measurements vector.

As can be seen in the above equations, attitude prediction through gyroscope measurements requires an integration, this makes that attitude predictions accumulate error. So an aided attitude estimation is implemented.

Aiding attitude measurements are derived from accelerometer and magnetometer measurements:

$$
\boldsymbol{q}_{(k)}^{a, m}=\boldsymbol{q}_{(k)}^{\psi} \otimes \boldsymbol{q}_{(k)}^{\theta} \otimes \boldsymbol{q}_{(k)}^{\phi}
$$

where; $\boldsymbol{q}_{(k)}^{a, m}$ is the quaternion attitude estimation from accelerometer and magnetometer measurements, $\boldsymbol{q}_{(k)}^{\psi}$ is the yaw attitude estimated from magnetometer measurements and $\boldsymbol{q}_{(k)}^{\theta}$ $\boldsymbol{q}_{(k)}^{\phi}$ are the pitch and roll attitude estimated from accelerometer measurements.

The accelerometer measurements based attitude estimations are computed as:

$$
\boldsymbol{q}_{(k)}^{\theta}=\left[\cos \left(\frac{\theta}{2}\right) \quad 0 \quad \operatorname{sen}\left(\frac{\theta}{2}\right) \quad 0\right]
$$

$$
\begin{aligned}
\boldsymbol{V}_{A U V}^{(k+1)} & =\left[\begin{array}{lll}
\sum_{j=1}^{N_{v}} w_{N j}^{(k+1)} v_{N j}^{(k+1)} & \sum_{j=1}^{N_{v}} w_{E j}^{(k+1)} v_{E j}^{(k+1)} & \sum_{j=1}^{N_{v}} w_{D j}^{(k+1)} v_{D j}^{(k+1)}
\end{array}\right] \\
\boldsymbol{P}_{A U V}^{(k+1)} & =\left[\begin{array}{lll}
\sum_{j=1}^{N_{p}} w_{L j}^{(k+1)} L_{j}^{(k+1)} & \sum_{j=1}^{N_{p}} w_{l j}^{(k+1)} l_{j}^{(k+1)} & \sum_{j=1}^{N_{p}} w_{z j}^{(k+1)} z_{j}^{(k+1)}
\end{array}\right]
\end{aligned}
$$




$$
\begin{gathered}
\cos \left(\frac{\theta}{2}\right)=\sqrt{\frac{1+\sqrt{1-\left(\overline{a_{X}}\right)^{2}}}{2}} \\
\operatorname{sen}\left(\frac{\theta}{2}\right)=\operatorname{sig}\left(\overline{a_{X}}\right) \sqrt{\frac{1-\sqrt{1-\left(\overline{a_{X}}\right)^{2}}}{2}}
\end{gathered}
$$

where; $\theta$ is the pitch angle ( $\mathrm{rad}), \overline{a_{X}}$ is the normalized $\mathrm{X}$ axis accelerometer measurement and $\operatorname{sig}()$ denotes the sign function.

$$
\begin{aligned}
& \boldsymbol{q}_{(k)}^{\phi}=\left[\cos \left(\frac{\phi}{2}\right) \quad \operatorname{sen}\left(\frac{\phi}{2}\right) \quad 0 \quad 0\right] \\
& \cos \left(\frac{\phi}{2}\right)=\sqrt{\frac{1+\sqrt{1-\left(\overline{a_{Y}}\right)^{2}}}{2}} \\
& \operatorname{sen}\left(\frac{\phi}{2}\right)=\operatorname{sig}\left(\overline{a_{Y}}\right) \sqrt{\frac{1-\sqrt{1-\left(\overline{a_{Y}}\right)^{2}}}{2}}
\end{aligned}
$$

where; $\phi$ is the roll angle (rad) and $\overline{a_{Y}}$ is the normalized $\mathrm{Y}$ axis accelerometer measurement.

$$
\begin{aligned}
& \boldsymbol{q}_{(k)}^{\psi}=\left[\cos \left(\frac{\psi}{2}\right) \quad 0 \quad 0 \quad \operatorname{sen}\left(\frac{\psi}{2}\right)\right] \\
& \cos \left(\frac{\psi}{2}\right)=\left\{\begin{array}{l}
\frac{\sqrt{m_{X Y c}^{E}+m_{X c}^{E} \sqrt{m_{X Y c}^{E}}}}{\sqrt{2 m_{X Y c}^{E}}} \text { if } m_{X c}^{E} \geq 0 \\
\frac{m_{Y c}^{E}}{\sqrt{2\left(m_{X Y c}^{E}-m_{X c}^{E} \sqrt{\left.m_{X Y c}^{E}\right)}\right.}} \text { if } m_{X c}^{E}<0
\end{array}\right. \\
& \operatorname{sen}\left(\frac{\psi}{2}\right)=\left\{\begin{array}{l}
\frac{m_{Y c}^{E}}{\sqrt{2\left(m_{X Y c}^{E}+m_{X c}^{E} \sqrt{\left.m_{X Y c}^{E}\right)}\right.}} \text { if } m_{X c}^{E} \geq 0 \\
\frac{\sqrt{m_{X Y c}^{E}-m_{X c}^{E} \sqrt{m_{X Y c}^{E}}}}{\sqrt{2 m_{X Y c}^{E}}} \text { if } m_{X c}^{E}<0
\end{array}\right.
\end{aligned}
$$

where; $\psi$ is the yaw angle (rad), $m_{X c}^{E}$ and $m_{Y c}^{E}$ are the magnetometer measurements calibrated and compensated for pitch and roll and $m_{X Y C}^{E}=\left(m_{X c}^{E}\right)^{2}+\left(m_{Y c}^{E}\right)^{2}$.

The calibrated magnetometer measurements are compensated for pitch and roll by a DCM:

$$
\boldsymbol{m}^{E}=\boldsymbol{C}_{b}^{n}\left(\boldsymbol{q}_{(k)}^{a}\right) \boldsymbol{m}_{c}
$$

where; $\boldsymbol{m}^{E}$ is the calibrated and pitch and roll compensated magnetometer measurements vector, $\boldsymbol{m}_{c}$ is the calibrated magnetometer measurements vector and $\boldsymbol{C}_{b}^{n}\left(\boldsymbol{q}_{(k)}^{a}\right)$ is the DCM evaluated for the pitch and roll orientation $\boldsymbol{q}_{(k)}^{a}$ estimated from the accelerometer measurements.

$$
\boldsymbol{q}_{(k)}^{a}=\boldsymbol{q}_{(k)}^{\theta} \otimes \boldsymbol{q}_{(k)}^{\phi}=\left[\begin{array}{llll}
q_{W a} & q_{X a} & q_{Y a} & q_{Z a}
\end{array}\right]
$$

The difference between the aiding attitude from the accelerometer and magnetometer measurements the attitude prediction from the gyroscope measurements provides an evaluation measurement to estimate an improved attitude estimation.

$$
\boldsymbol{\delta}_{(k)}=\boldsymbol{q}_{(k)}^{a, m}-\boldsymbol{q}_{(k)}^{g}=\left[\begin{array}{llll}
\delta_{W} & \delta_{X} & \delta_{Y} & \delta_{Z}
\end{array}\right]_{(k)}
$$

A PF based attitude estimation algorithm based in the previous equations was implemented. The PF algorithm stages for the attitude estimation are:

Initialization: a set of $N$ particles representing probable attitude states is generated and an equal weight is assigned to every particle component.

$$
\begin{gathered}
\boldsymbol{q}_{i}^{0}=\left[\begin{array}{cccc}
q_{i W}^{0} & q_{i X}^{0} & q_{i Y}^{0} & q_{i Z}^{0}
\end{array}\right] \text { with } i=1, \ldots, N \\
\boldsymbol{w}_{i}^{0}=\left[\begin{array}{llll}
\frac{1}{N} & \frac{1}{N} & \frac{1}{N} & \frac{1}{N}
\end{array}\right] \forall i
\end{gathered}
$$

Prediction: when a gyroscope measurement is available, the particle set is propagated according to equation (28).

Update: when both accelerometer and magnetometer are available, the aiding attitude is estimated and the $\boldsymbol{\delta}_{(k)}$ is calculated for every particle. The particles weights are then updated through a likelihood function:

$$
w_{i j}^{k+1}=\frac{e^{\frac{-\left(\delta_{i j}^{k}\right)^{2}}{2 \sigma_{\delta}^{2}}}}{\sigma_{\delta} \sqrt{2 \pi}} w_{i j}^{k}
$$

where; $\sigma_{\delta}$ is the component attitude difference standard deviation, sub-indexes $i$ and $j$ correspond to the particle number and component respectively. Then the updated weights are normalized.

Re-sampling: after weights update, the effective particle number is calculated for each particle component $j$ to determinate if the re-sampling process must be executed:

$$
N_{E F j}^{k+1}=\frac{1}{\sum_{i=1}^{N}\left(w_{i j}^{k+1}\right)^{2}}
$$

If $N_{E F j}^{k+1}$ is lower than a experimentally determined threshold $N_{T H}$ the selection and replace re-sampling algorithm is applied [25]. Then, the weights of the re-sampled components are updated assigning to all the same and equal to $1 / N$.

Estimation: the attitude is estimated as the weighted mean of al particles components (see equation (47)).

\section{2) Position estimation}

In this work the AUV position is expressed in terms of depth and coordinates in longitude and latitude. Depth is assumed known and measured by a specific sensor onboard the AUV [26].

The horizontal position (longitude and latitude) when the AUV is in surface or at very low depth $(<4 \mathrm{~m})$ is obtained by a GPS receiver. In other case, a PF based estimation algorithm is applied using the time of flight (TOF) measurements from communications between a known position platform and the AUV and the velocity estimations provided by the AUV navigation system. 
In this work is assumed that the known position platform is a surface buoy. The buoy and the AUV are equipped with acoustic modems and a positioning message is broadcasted by the buoy every 30 seconds. In this message, the buoy position is part of the data, so every time the AUV receives the positioning message, the TOF is estimated and the buoy position is obtained. With the TOF measurement and sound speed information of the operation area, the AUV estimates the distance between its actual position and the buoy position.

A PF based estimation algorithm was developed with this information to obtain an improved estimation of the AUV position compared to the one obtained by the single integration of the velocity data of the AUV navigation system. The stages of the developed PF based algorithm are described below:

Initialization: the PF particles represents probable AUV positions. The particle set $\boldsymbol{S}_{j}^{0}$ is initialized around the last known AUV position $\left(L_{A U V}^{0}, l_{A U V}^{0}\right)$ such as the last GPS measurement before the AUV dives in. $\boldsymbol{S}_{j}^{0}$ consists of $N$ particles uniformly distributed an all with the same weight $1 / N$.

$$
\begin{gathered}
\boldsymbol{S}_{j}^{0}=\left[\begin{array}{ll}
L_{j}^{0} & l_{j}^{0}
\end{array}\right] \text { with } j=1, \ldots, N \\
w_{j}^{0}=\frac{1}{N} \forall j
\end{gathered}
$$

Prediction: if in an instant $k$ data from the AUV navigation system velocity estimation $v_{N A U V}^{k}$ and $v_{E A U V}^{k}$ are available the particles are propagated according to equation (50).

where; $T_{S}$ is the navigation system update period (s), $z_{A U V}^{k}$ is the AUV depth measurement $(\mathrm{m})$ and $R$ is the Earth radius (6378137 m).

Update: if at the instant $k$ a communication from the buoy is received and through the TOF measurement the distance $d^{k}$ between the surface buoy and the AUV is estimated. The distances between the particles positions $\left(L_{j}^{k}, l_{j}^{k}\right)$ and the known buoy position $\left(L_{B}^{k}, l_{B}^{k}\right)$ considering the AUV depth $z_{A U V}^{k}$ are calculated.

$$
d_{j}^{k}=\sqrt{\left(d_{j B}^{k}\right)^{2}+\left(z_{A U V}^{k}-z_{B}\right)^{2}} \text { for } j=1 \text { to } N
$$

where; $z_{B}$ is the depth (m) of the acoustic modem installed in the buoy (also a known value) and $d_{j B}^{k}$ is the distance (m) at the instant $k$ between the position of the particle $j$ and the position of the surface buoy, see equations (51) and (52) where;

$L_{B}^{k}$ and $l_{B}^{k}$ are the latitude and longitude positions of the Surface buoy at the instant $k$ while $L_{j}^{k}$ and $l_{j}^{k}$ are the latitude and longitude positions of the $j$ particle at the instant $k$.

The particles weights are updated through a likelihood function and the difference between the distance between the AUV and the surface buoy estimated with the communication TOF measurement and the distances between the particles positions and the position of the surface buoy.

$$
w_{j}^{k+1}=\frac{e^{\frac{-\left(d_{j}^{k}-d^{k}\right)^{2}}{2 \sigma_{d}^{2}}}}{\sigma_{d} \sqrt{2 \pi}} w_{j}^{k} \text { for } j=1 \text { to } N
$$

where; $\sigma_{d}$ is the distance difference standard deviation. Then the updated weights are normalized.

Re-sampling: this stage is performed if the effective number of particles $N_{E F}^{k+1}$ is lower than a threshold $N_{T H}$ (see equation (11)).

If this condition is satisfied there will be $\left(N-N_{T H}\right)$ (with $N$ not determined yet) particles in the set with a negligible weight, in other words, those particles represent a state with a very low probability of represent the real state of the system.

First, the equivalent number of particles $N_{E Q j}^{k+1}$ represented by the particles weights are calculated:

$$
N_{E Q j}^{k+1}=N w_{j}^{k+1} \text { for } j=1 \text { to } N
$$

Then a new set of particles is generated by reproducing $N_{E Q j}^{k+1}$ times the particle $j$. In this way only the particles with a significant probability of represent the real state of the system are reproduced. This new set will contain less than $N$ particles. The set is completed with particles uniformly distributed around the particle with higher weight, allowing the exploration of the state space around the best estimation of the set at the moment. After the re-sampling all the particles weights are set to $1 / N$.

Estimation: two cases are distinguished in the estimation step. If the re-sampling stage wasn't executed the state estimation $\boldsymbol{S}_{P S}^{k+1}=\left[\begin{array}{ll}L_{P S}^{k+1} & l_{P S}^{k+1}\end{array}\right]$ is obtained as the weighted mean of the particle set

$$
\boldsymbol{S}_{P S}^{k+1}=\sum_{j=1}^{N} \boldsymbol{S}_{j}^{k+1} w_{j}^{k+1}
$$

$$
\begin{gathered}
\boldsymbol{q}_{P S}^{(k+1)=}\left[\sum_{i=1}^{N} w_{i W}^{(k+1)} q_{i W}^{(k+1)} \sum_{i=1}^{N} w_{i X}^{(k+1)} q_{i X}^{(k+1)} \sum_{i=1}^{N} w_{i Y}^{(k+1)} q_{i Y}^{(k+1)} \sum_{i=1}^{N} w_{i Z}^{(k+1)} q_{i Z}^{(k+1)}\right] \\
\boldsymbol{S}_{j}^{k+1}=\boldsymbol{S}_{j}^{k}+T_{S}\left[\frac{v_{N A U V}^{k}}{\left(R+z_{A U V}^{k}\right)}, \frac{v_{E A U V}^{k}}{\cos \left(L_{j}^{k}\right)\left(R+z_{A U V}^{k}\right)}\right] \text { for } j=1 \text { to } N \\
d_{j B}^{k}=2 R\left[\operatorname{atan} 2\left(\sqrt{a_{j}^{k}}, \sqrt{1-a_{j}^{k}}\right)\right] \text { for } j=1 \text { to } N \\
a_{j}^{k}=\operatorname{sen}^{2}\left(\frac{L_{B}^{k}-L_{j}^{k}}{2}\right)+\cos \left(L_{B}^{k}\right) \cos \left(L_{j}^{k}\right) \operatorname{sen}^{2}\left(\frac{l_{B}^{k}-l_{j}^{k}}{2}\right) \text { for } j=1 \text { to } N
\end{gathered}
$$


If the re-sampling stage was executed, the state estimation is performed as the weighted mean of the particles that were reproduced, excluding those that were generated to complete the set, since that those were not updated yet. In this case, the sum of the weights of the reproduced particles is calculated:

$$
w_{R M}^{k+1}=\sum_{j=1}^{M} w_{j}^{k+1}
$$

where; $M$ is the number of particles reproduced from the set previous to the re-sampling. Then the estate estimation is obtained as the weighted mean of the sub-set of $M$ particles, normalizing its weights with $w_{R M}^{k+1}$ :

$$
\boldsymbol{S}_{P S}^{k+1}=\sum_{j=1}^{M} \frac{\boldsymbol{S}_{j}^{k+1} w_{j}^{k+1}}{w_{R M}^{k+1}}
$$

At this point, navigation and positioning solutions were detailed with focus in the details of the estimation algorithms applied.

\section{IMPLEMENTATION AND PRELIMINARY RESULTS}

Considering that the developed solutions are intended to be integrated into an AUV the algorithms implementation was coded in $\mathrm{C}++$ language and supported by the Robot Operating System (ROS), to take advantage of the development and integration benefits that this system provides [27]. All systems were implemented as ROS packages and integrated in a simulation architecture that emulates the AUV dynamics, guidance and control systems and onboard sensors, also a surface buoy was simulated as a separated package and acoustic communications cycles between buoy and AUV are also considered. Next the preliminary results of the performance of the developed systems are presented

\section{A. Attitude Estimation}

Attitude estimation algorithm developed in Section III-B-1 was implemented as a ROS node. A Xsens MTi-30-2A5G4 IMU [28] was used to acquire gyroscope, accelerometer and magnetometer measurements at a sampling rate of $100 \mathrm{~Hz}$.

Prior to execute the estimation algorithm a calibration step is performed. During calibration the IMU is maintained static and leveled. The accelerometer and gyroscopes axis offsets are calculated as the average of 400 measurements. Magnetometer calibration is then performed, but due to the local magnetic field perturbations, named soft-iron and hard-iron, a more complex calibration procedure is required. In this work a Particle Swarm Optimization (PSO) algorithm was implemented [29]. In a calibrated magnetometer the measurements locus is a centered sphere, while in an uncalibrated magnetometer the locus is a non-centered spheroid. During the magnetometer calibration a set of measurements moving the IMU in different directions is acquired and through the PSO the equivalent spheroid parameters are estimated to correct the uncalibrated measurements. In Fig. 2 an example of the magnetometer calibration is illustrated.
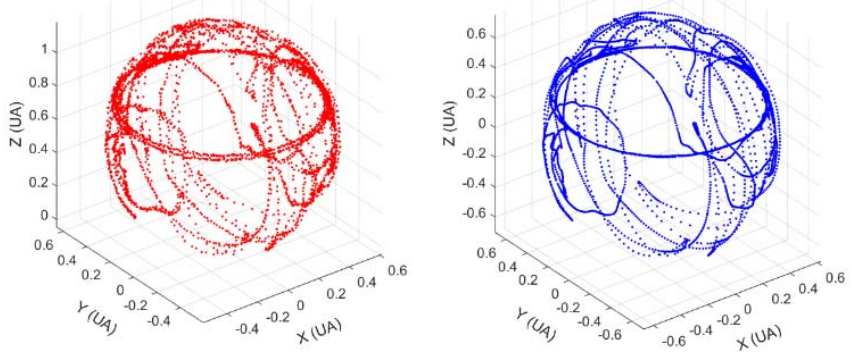

Fig. 2. Uncalibrated magnetometer measurements (left) and PSO calibrated measurements (right).

Once the calibration step is performed the PF for attitude estimation is executed.

Performance evaluation was carried out by analyzing three IMU conditions; static, pitch and roll movements and yaw movement.

The static condition was performed to evaluate the long term stability of the estimate and verify if drift occurs. While pitch, roll and yaw movements were performed to evaluate the short term estimation quality. In Figures 3, 4 and 5 the attitude estimation results for the IMU proprietary attitude output and the PF estimation for each movement condition are shown.


Fig. 3. IMU attitude estimation in static position; propetary IMU algorithm (left) and PF estimation algorithm (right).
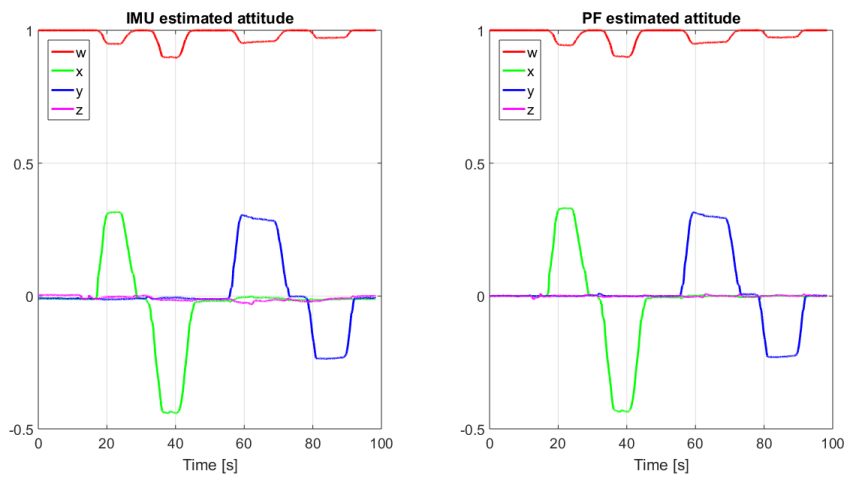

Fig. 4. IMU attitude estimation under pitch and roll movements; propetary IMU algorithm (left) and PF estimation algorithm (right). 

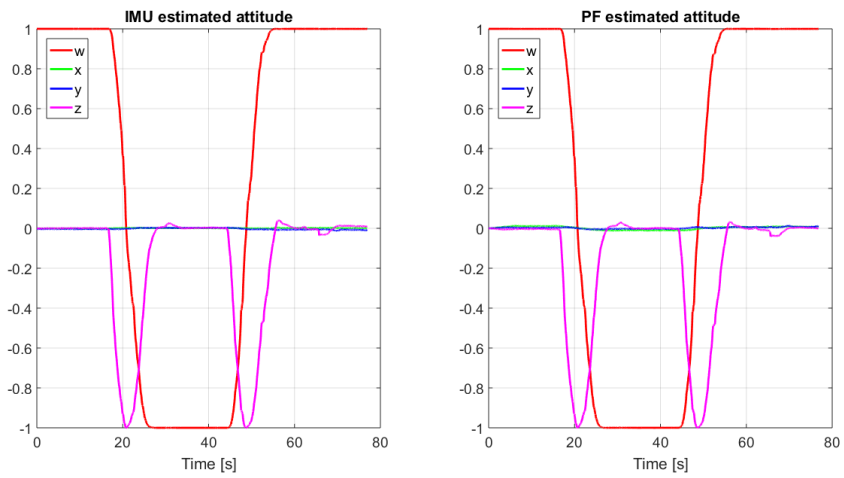

Fig. 5. IMU attitude estimation under yaw-movement; propetary IMU algorithm (left) and PF estimation algori , , ht).

For each movement condition 10 tests were performed to obtain the Root Mean Square (RMS) attitude estimation error. Comparison of the gyroscope attitude estimation (equation (28)), the IMU proprietary attitude output and the PF estimation were performed against the aiding measurements to obtain a common error figure. In Table I the results for each condition are presented. In every case the results obtained with the PF are superior to those of the gyroscope integration and the proprietary IMU attitude output.

\section{B. Navigation System}

The positioning and navigation solutions were implemented as ROS packages configuring a complete AUV navigation system. The NS performance was evaluated in a ROS based simulation environment. An AUV was emulated by means of a dynamic model, guidance and control systems were also implemented to perform parallel transects mission profiles (adequate for acoustic survey applications). Onboard AUV sensors such as IMU, GPS, depth meter, DVL and acoustic link to a surface buoy with GPS are also simulated considering technical specifications of commercial devices.

Results of velocity and position errors for the INS computation, DVL aided INS and the developed NS against the real AUV velocity and position were computed in a real time like mission execution.

In Fig. 6 the DVL aided INS, the developed NS and the true AUV trajectories are shown, in this case INS trajectory was omitted due to its large deviation from the true trajectory.

Its noted that the developed NS provides a better position estimate than the DVL aided INS that shows a cumulative trend in position error. This is clearly seen in Fig. 7 were errors for horizontal and vertical position are shown. For comparison propose only in Fig. 8 the position error for the INS computation without aiding is shown.

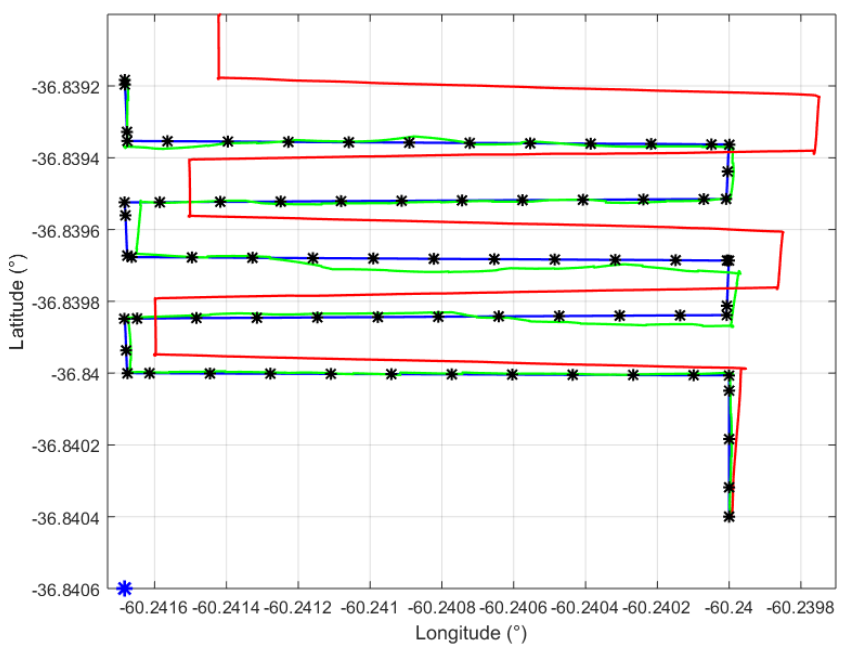

Fig. 6. DVL aided INS (red), the developed NS (green) and true AUV trajectories for a five legs parallel mission profile. Blue asterisk indicates the surface buoy position and the black asterisks indicates an acoustic communication event, thus a range aideding update.
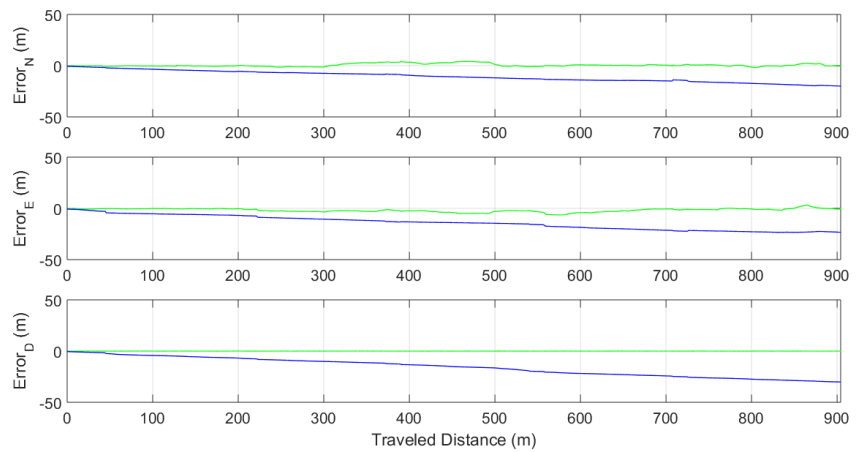

Fig. 7. Position errors for North, East and Down directions for the DVL aided INS (blue) and the developed NS (green).
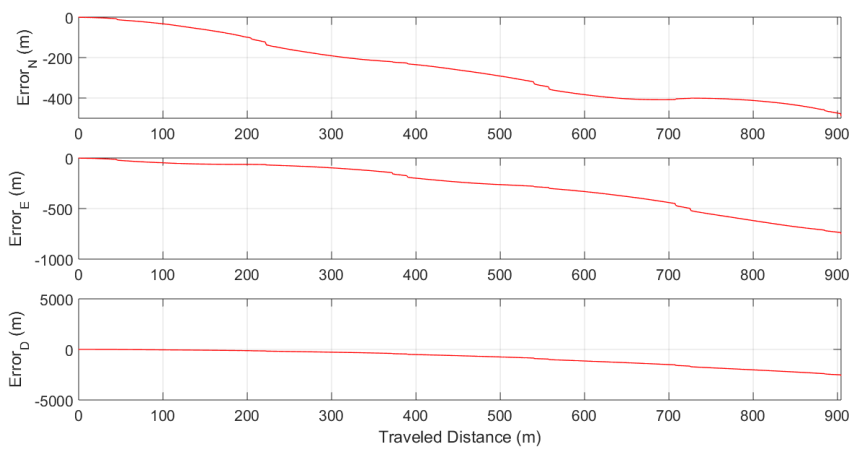

Fig. 8. Position errors for North, East and Down directions for the unaided INS computation.

TABLE I. IMU ATTITUDE RMS ESTIMATION ERRORS

\begin{tabular}{|c|c|c|c|c|c|c|c|c|c|}
\hline \multirow{2}{*}{$\begin{array}{c}\text { Attitude } \\
\text { component }\end{array}$} & \multicolumn{3}{|c|}{$\begin{array}{c}\text { Gyroscope } \\
\text { Integration }\end{array}$} & \multicolumn{3}{c|}{$\begin{array}{c}\text { IMU } \\
\text { estimation }\end{array}$} & \multicolumn{3}{c|}{$\begin{array}{c}\text { PF } \\
\text { estimation }\end{array}$} \\
\cline { 2 - 11 } & Static & $\begin{array}{c}\text { Pitch } \\
\text { and roll }\end{array}$ & Yaw & Static & $\begin{array}{c}\text { Pitch } \\
\text { and roll }\end{array}$ & Yaw & Static & $\begin{array}{c}\text { Pitch } \\
\text { and roll }\end{array}$ & Yaw \\
\hline$w$ & $3.7 \mathrm{E}-3$ & $1.8 \mathrm{E}-3$ & $1.3 \mathrm{E}-2$ & $3.4 \mathrm{E}-5$ & $1.9 \mathrm{E}-3$ & $1.5 \mathrm{E}-2$ & $2.7 \mathrm{E}-5$ & $4.5 \mathrm{E}-4$ & $1 \mathrm{E}-2$ \\
\hline$x$ & $1.1 \mathrm{E}-2$ & $4.1 \mathrm{E}-3$ & $3.5 \mathrm{E}-2$ & $3.6 \mathrm{E}-3$ & $1.2 \mathrm{E}-2$ & $9.6 \mathrm{E}-3$ & $5.3 \mathrm{E}-4$ & $1.5 \mathrm{E}-3$ & $3.4 \mathrm{E}-3$ \\
\hline$y$ & $7.3 \mathrm{E}-3$ & $8.1 \mathrm{E}-3$ & $2.9 \mathrm{E}-2$ & $6.4 \mathrm{E}-3$ & $9.6 \mathrm{E}-3$ & $9.7 \mathrm{E}-3$ & $5.5 \mathrm{E}-4$ & $1.4 \mathrm{E}-3$ & $4 \mathrm{E}-3$ \\
\hline$z$ & $1.9 \mathrm{E}-2$ & $1.2 \mathrm{E}-2$ & $5.9 \mathrm{E}-2$ & $4.1 \mathrm{E}-3$ & $1.3 \mathrm{E}-2$ & $1.1 \mathrm{E}-2$ & $3.9 \mathrm{E}-4$ & $2.2 \mathrm{E}-3$ & $7.2 \mathrm{E}-3$ \\
\hline
\end{tabular}


In Fig. 9 the velocity estimate for the developed NS is compared with the true AUV velocity.

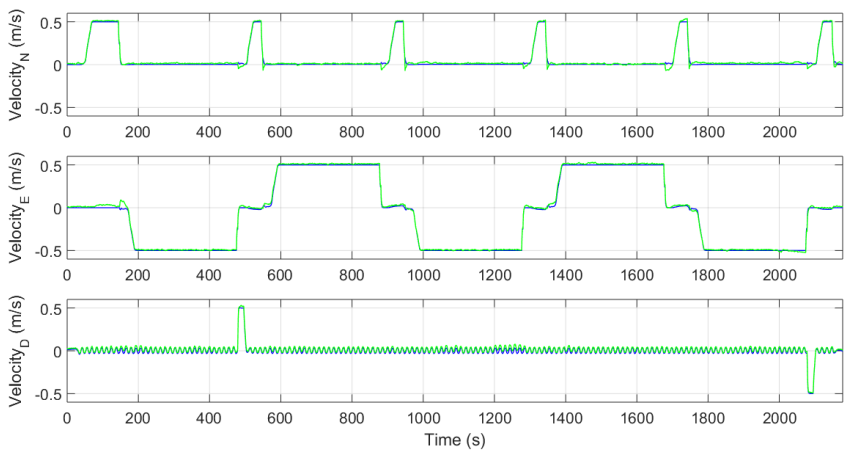

Fig. 9. AUV true velocity in the North, East and Donwn directions (blue) and the corresponding estimations from the developed NS (blue)..

From these results the following error figures were extracted; for the DVL aided INS position error shows a tendency to accumulate linearly with the traveled distance with errors of 23.8, 29 and $32.8 \mathrm{~m} / \mathrm{km}$ for North, East and Down direction respectively. While the developed NS shows an oscillatory error with RMS values of 1.54, 2.38 and $0.03 \mathrm{~m}$ for the North, East and Down directions respectively. Velocity errors for both the DVL aided INS and the developed NS are the same since the first is the output of the velocity PF and in RMS values are $0.014,0.016$ and $0.016 \mathrm{~m} / \mathrm{s}$ for the North, East and Down directions respectively

\section{CONCLUSSIONS}

In this work the development of an underwater navigation system is presented. The system is based on a DVL and range from a surface buoy aided INS. Extensive use of PF estimation algorithm were applied. Results from simulations shows a very good performance of the system. Future work will consist in the simulation of the system replacing the surface buoy by a surface vehicle that develops trajectories to benefit the performance of the range aiding. Also an attitude estimation algorithm applying a PSO for magnetometer calibration and PF for estimation is presented. The performance from experimental tests of attitude estimation are encouraging outperforming the IMU proprietary attitude output. Implementation of the systems as ROS packages considers its integration in a real AUV.

\section{REFERENCES}

[1] M. Choyekh et al., "Vertical water column survey in the Gulf of Mexico using autonomous underwater vehicle SOTAB-I," Journal of Marine Technology Society, vol. 49, no. 3, pp. 88-101, 2015.

[2] J. Jamieson and L. Wilson, "Effective Launch and Recovery of Autonomous Underwater Vehicles," Offshore Technology Conference, no. May, pp. 1-14, 2015.

[3] [8] S. Sariel, T. Balch, and N. Erdogan, "Naval Mine Countermeasure Missions," IEEE Robotics \& Automation Magazine, pp. 45-52, 2008.

[4] T. I. Fossen, Marine Control Systems Guidance, Navigation, and Control of Ships, Rigs and Underwater Vehicles, Marine Cybernetics, Trondheim, Norway, 2002.

[5] I. F. Akyildiz, D. Pompili and T. Melodia, "Underwater acoustic sensor networks: research challenges," Journal of Ad Hoc Networks, vol. 3, no. 3, pp. 257-279, 2005.

[6] K. Vickery, "Acoustic positioning systems. A practical overview of current systems," Proceedings of the 1998 Workshop on Autonomous Underwater Vehicles, Cambridge, MA, USA, pp. 5-17, 1998.
[7] A. Alcocer, P. Oliveira and A. Pascoal, "Underwater Acoustic Positioning Systems Based on Buoys with GPS," Proceedings of the Eighth European Conference on Underwater Acoustics, 8th ECUA, pp. 1-8, 2006.

[8] D. Rudolph and T. A. Wilson, "Doppler Velocity Log theory and preliminary considerations for design and construction," Proceedings of IEEE Southeastcon, Orlando, FL., pp. 1-7, 2012.

[9] C. Meurer, J. Francisco Fuentes-Pérez, N. Palomeras, M. Carreras and M. Kruusmaa, "Differential Pressure Sensor Speedometer for Autonomous Underwater Vehicle Velocity Estimation," IEEE Journal of Oceanic Engineering, pp. 1-33.

[10] P. Ridao, D. Ribas, E. Hernàndez and A. Rusu, "USBL/DVL navigation through delayed position fixes," IEEE International Conference on Robotics and Automation, Shanghai, pp. 2344-2349, 2011.

[11] D. H. Titterton and J. L. Weston, "Strapdown inertial navigation technology", Institution of Electrical Engineers, 2004.

[12] D. B. Marco and A. J. Healey, "Command, control, and navigation experimental results with the NPS ARIES AUV," IEEE Journal of Oceanic Engineering, vol. 26, no. 4, pp. 466-476, 2001.

[13] S. Thrun, W. Burgard and D. Fox, "Probabilistic Robotics", The MIT Press, 2005.

[14] P. A. Miller, J. A. Farrell, Y. Zhao and V. Djapic, "Autonomous Underwater Vehicle Navigation," IEEE Journal of Oceanic Engineering, vol. 35, no. 3, pp. 663-678, 2010.

[15] J. H. Kepper, B. C. Claus and J. C. Kinsey, "MEMS IMU and one-waytravel-time navigation for autonomous underwater vehicles," OCEANS 2017 - Aberdeen, Aberdeen, pp. 1-9, 2017.

[16] P. Liu, B. Wang, Z. Deng and M. Fu, "INS/DVL/PS Tightly Coupled Underwater Navigation Method with Limited DVL Measurements," IEEE Sensors Journal, vol. 18, no. 7, pp. 2994-3002, 2018.

[17] B. Allotta, R. Constanzi, F. Fanelli, D. Fenucci, E. Meli and A. Ridolfi, "A new AUV navigation system exploiting unscented Kalman filter," Ocean Engineering, vol. 113, pp. 1321-132, 2016.

[18] M. S. Arulampalam, S. Maskell, N. Gordon and T. Clapp, "A tutorial on particle filters for online nonlinear/non-Gaussian Bayesian tracking," IEEE Transactions on Signal Processing, vol. 50, no. 2, pp. 174-188, 2002.

[19] F. S. Dubrovin and A. F. Scherbatyuk, "Studying some algorithms for AUV navigation using a single beacon: The results of simulation and sea trials," Gyroscopy and Navigation, vol. 7, no. 2, pp. 189-196, 2016.

[20] G. T. Donovan, "Position Error Correction for an Autonomous Underwater Vehicle Inertial Navigation System (INS) Using a Particle Filter," IEEE Journal of Oceanic Engineering, vol. 37, no. 3, pp. 431-445, 2012

[21] I. Y. Bar-itzhack and K. A. Fegley, "Orthogonalization Techniques of a Direction Cosine Matrix," IEEE Transactions on Aerospace and Electronic Systems, vol. 5, no. 5, pp. 798-804, 1969.

[22] M. Bolić, P. M. Djurić and S. Hong, "Resampling Algorithms for Particle Filters: A Computational Complexity Perspective," EURASIP Journal on Applied Signal Processing, pp. 2267-2277, 2004.

[23] T. Li, M. Bolić and P. M. Djurić, "Resampling Methods for Particle Filtering: Classification, implementation, and strategies," IEEE Signal Processing Magazine, vol. 32, no. 3, pp. 70-86, 2015.

[24] B. Ristic, S. Arulampalam and N. Gordon, "Beyond the Kalman Filter; Particle Filters for Tracking Applications," Artech House, Boston, Ma., 2004.

[25] I. M. Rekleitis, "A particle filter tutorial for mobile robot localization," Center for Intelligent Machines Technical Report TR-CIM-04-02, McGill University, Montreal, Quebec, Canada, 2002.

[26] ImpactSubsea ISD4000 depth sensor specifications, 2019.

[27] A. Koubaa, Robot Operating System (ROS) The Complete Reference, 1st. Ed., vol. 1. Springer International Publishing, 2016.

[28] Xsens Technologies, "MTi 10 and MTi 100 series Technical Specifications", 2019.

[29] J. Kennedy and R. Eberhart, "Particle Swarm Optimization," Proceedings of ICNN'95 - International Conference on Neural Networks, Perth, WA, Australia, vol. 4, pp. 1942-1948, 1995. 